ARTICLE

Received 8 Aug 2014 | Accepted 9 Jan 2015 | Published 20 Feb $2015 \quad$ DOl: 10.1038/ncomms7248

\title{
Crystalline silicon core fibres from aluminium core preforms
}

Chong Hou ${ }^{1,2,3}$, Xiaoting Jia ${ }^{2,3}$, Lei Wei ${ }^{4}$, Swee-Ching Tan ${ }^{5}$, Xin Zhao ${ }^{1}$, John D. Joannopoulos ${ }^{2,3,6} \&$ Yoel Fink ${ }^{1,2,3,7}$

Traditional fibre-optic drawing involves a thermally mediated geometric scaling where both the fibre materials and their relative positions are identical to those found in the fibre preform. To date, all thermally drawn fibres are limited to the preform composition and geometry. Here, we fabricate a metre-long crystalline silicon-core, silica-cladded fibre from a preform that does not contain any elemental silicon. An aluminium rod is inserted into a macroscopic silica tube and then thermally drawn. The aluminium atoms initially in the core reduce the silica, to produce silicon atoms and aluminium oxide molecules. The silicon atoms diffuse into the core, forming a large phase-separated molten silicon domain that is drawn into the crystalline silicon core fibre. The ability to produce crystalline silicon core fibre out of inexpensive aluminium and silica could pave the way for a simple and scalable method of incorporating silicon-based electronics and photonics into fibres.

\footnotetext{
${ }^{1}$ Department of Materials Science and Engineering, Massachusetts Institute of Technology, Cambridge, Massachusetts 02139, USA. ${ }^{2}$ Institute of Soldier Nanotechnology, Massachusetts Institute of Technology, Cambridge, Massachusetts 02139, USA. ${ }^{3}$ Research Laboratory of Electronics, Massachusetts Institute of Technology, Cambridge, Massachusetts 02139, USA. ${ }^{4}$ School of Electrical and Electronic Engineering, Nanyang Technological University, 50 Nanyang Avenue, Singapore 639798, Singapore. ${ }^{5}$ Department of Materials Science and Engineering, National University of Singapore, 9 Engineering Drive 1, Singapore 117575, Singapore. ${ }^{6}$ Department of Physics, Massachusetts Institute of Technology, Cambridge, Massachusetts 02139, USA. ${ }^{7}$ Department of Electrical Engineering and Computer Science, Massachusetts Institute of Technology, Cambridge, Massachusetts 02139, USA. Correspondence and requests for materials should be addressed to Y.F. (email: yoel@mit.edu).
} 
S ilicon $(\mathrm{Si})$ and its oxide silica $\left(\mathrm{SiO}_{2}\right)$ have both played an important role in modern day electronics and communications ${ }^{1-10}$. In recent years the opportunities for tighter integration between electronics and photonics have led to the emergence of the field of Si photonics where wafer processes are used to combine the best features of both materials ${ }^{11-14}$. In a mirror effort the advanced demonstrations of incorporating $\mathrm{Si}$ into fibres have been explored in electronics and photonics, such as photodetection ${ }^{15}$, fibre battery ${ }^{16}$, fibre solar cell ${ }^{17,18}$, electro-optical modulation ${ }^{19}$, material engineering ${ }^{20}$, non-linear optics $^{21,22}$, pressurized Si sphere fabrication ${ }^{23}$ and so on. Two disparate methods of producing Si-core silica-cladded fibres have been reported, the first relies on the physical flow process and the second on a chemical reaction internal to a pre-fabricated fibre. In the first method, high-purity $\mathrm{Si}$ is placed inside a silica preform and drawn down into a silica fibre with a molten $\mathrm{Si}$ core ${ }^{23,24}$. The second involves the use of high-pressure $\mathrm{Si}$ precursor gas $\left(\mathrm{SiH}_{4}\right)$ to deposit $\mathrm{Si}$ inside a pre-fabricated hollow channel fibre via a chemical vapour deposition (CVD) process ${ }^{25}$. The CVD method is more versatile while the thermal drawing approach is more scalable.

The placement of disparate materials thermally drawn into close proximity at elevated temperatures presents compelling opportunities for inducing chemical reactions between the constituent materials ${ }^{26-28}$ that could in principle provide the versatility of chemical reactions with the scalability of fibre drawing. Results of this approach that we call fibre-draw synthesis to date have been confined to reactions in only very small domains at an interface between materials.

In this work, we utilize the redox reaction between aluminium $(\mathrm{Al})$ and silica $\left(\mathrm{SiO}_{2}\right)$ during fibre drawing to produce a fibre with a different core composition than that of the preform. This occurs through the following reaction:

$$
4 \mathrm{Al}+3 \mathrm{SiO}_{2} \rightarrow 2 \mathrm{Al}_{2} \mathrm{O}_{3}+3 \mathrm{Si}
$$

At high drawing temperature, the metallic $\mathrm{Al}$ reacts with the cladding material $\mathrm{SiO}_{2}$ and reduces the $\mathrm{Si}$. The products $\left(\mathrm{Al}_{2} \mathrm{O}_{3}\right.$ and $\mathrm{Si}$ ) then get phase separated, thus forming a pure Si-core in silica fibre. The reaction, diffusion and the phase separation all take place at an elevated temperature close to the fibre draw temperature.

\section{Results}

Fibre fabrication and characterization. The proposed method centres on a technique so-called preform-to-fibre fabrication by thermally drawing a macroscopic solid-state preform into extended lengths of uniform fibres, as shown in Fig. 1a. The preform consists of one piece of $\mathrm{Al}$ wire $(3 \mathrm{~cm}$ long, $250 \mu \mathrm{m}$ diameter) placed inside a silica tube (inner diameter $300 \mu \mathrm{m}$, outer diameter $600 \mu \mathrm{m}$ ). This assembly is further cladded with two concentric silica tubes (one with $1 \mathrm{~mm}$ inner diameter, $6 \mathrm{~mm}$ outer diameter, and the other one with $6 \mathrm{~mm}$ inner diameter and $12 \mathrm{~mm}$ outer diameter), as shown in Fig. 1b. The chamber where the $\mathrm{Al}$ wire resides is then pumped to $10^{-3}$ mbar and sealed with silica rods on both ends to prevent $\mathrm{Al}$ in contact with air. The preform with the $\mathrm{Al}$ core is then heated up to $2,200^{\circ} \mathrm{C}$ in Argon atmosphere and pulled into metre-long fibres with the diameters of $\sim 500 \mu \mathrm{m}$. During the fibre draw, Al melts and reacts with the surrounding silica cladding, and reduce Si atoms out of silica ${ }^{29}$. $\mathrm{Si}$ atoms accumulate during this process and finally occupy the entire core, resulting in a Si-core fibre with silica cladding, as shown in Fig. 1c.

Figure 2a shows an optical image of the fibre and indicates a change in core material as the fibre is drawn. From top to bottom, the colour of the fibre core is seen to transit from light yellow to black. Scanning electron microscope (SEM) and energy-dispersive

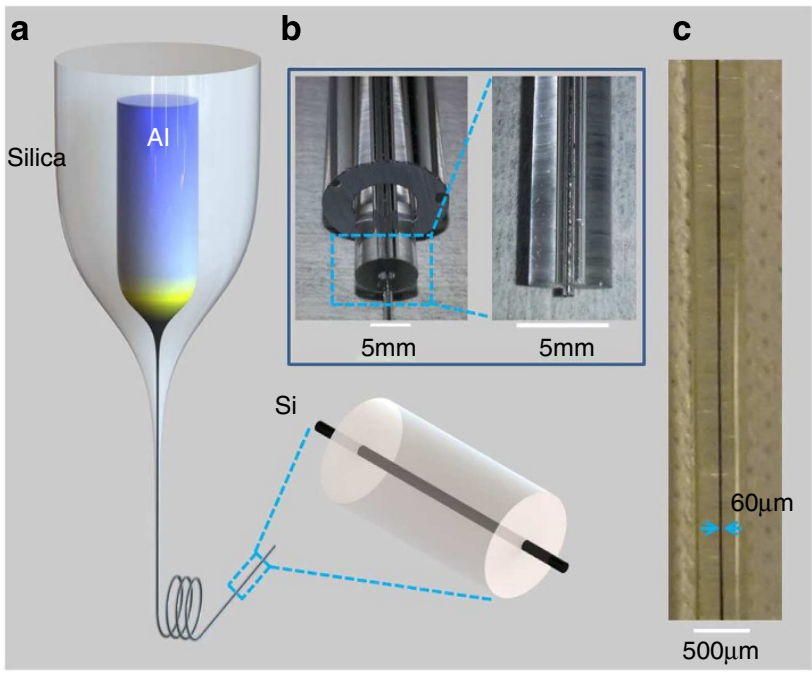

Figure 1 | Aluminium-core-silica-cladding preform drawn into silicon-core fibre. (a) The schematic diagram of the drawing process. A macroscopic preform with an elemental Al core surrounded with silica is thermally drawn into a fibre with an elemental $\mathrm{Si}$ core and a silica cladding. (b) The optical image of the preform before the shiny Al core is sealed in the cladding. (c) The optical image of the drawn fibre with a black Si core. The core diameter is about $60 \mu \mathrm{m}$.

X-ray spectroscopy (EDX) analyses are taken at four locations along the length of the fibre. Figure $2 b-e$ is SEM images of the cross-sections at locations 1-4 marked in Fig. 2a, respectively. Comparing these four figures, one notice there are light-grey dotswithin the core region in Fig. 2c-e. The grey dot in Fig. 2c is so small that is hardly noticed, but can be seen more clearly from EDX mapping on a zoomed-in area as shown in Fig. 2o. The dots in Fig. 2d,e are much larger. It is worth mentioning though that these dots in Fig. 2c-e, however, are quite different in forming mechanism and in $3 \mathrm{D}$ shape. In Fig. $2 \mathrm{c}$ the dot is actually a precipitated spherical drop composed of a majority of Si. But in Fig. $2 \mathrm{e}$ the dot is actually cross-section of the long crystalline $\mathrm{Si}$ wire (Supplementary Note 1). The EDX analyses around these areas are shown to the right of the four SEM images and confirm that the dots in locations \#3 and \#4 are composed of high-purity Si. In Fig. 2f-q, green, red and yellow colours denote element $\mathrm{Al}$, oxygen $(\mathrm{O})$ and $\mathrm{Si}$, respectively. In Fig. 2f,j, $\mathrm{n}$ (location \#1), the core is composed mainly of $\mathrm{Al}$ and $\mathrm{O}$. In Fig. 2g,k,o (location \#2), a small agglomeration of $\mathrm{Si}$ appears while $\mathrm{Al}$ and $\mathrm{O}$ still dominate the core area. In Fig. $2 \mathrm{~h}, \mathrm{l}, \mathrm{p}$ (location \#3), a large Si core is formed in the centre of the core area, surrounded by a thick layer composed of $\mathrm{Al}$ and $\mathrm{O}$. Finally, in Fig. 2i,m, (location \#4 which is in the black core section), $\mathrm{Si}$ occupies the entire core region, leaving $\mathrm{Al}$ to only a thin layer between the core and the cladding.

To further explore the differences between the light-yellow and black core regions, transmission electron microscopy (TEM)based analyses are performed at two different cross-sections (see Supplementary Fig. 1 for preparation of TEM samples), corresponding approximately to locations \#2 and \#4, respectively. In the core of a yellow-core region, we find that spherical drops form scattered along the axial direction (Supplementary Fig. 2). If we focus on one of these spherical drops, we obtain the results shown in the top panel of Fig. 3. Figure 3a shows that the sphere is about $100 \mathrm{~nm}$ in diameter and is surrounded by a circle of different materials. The diffraction pattern in Fig. $3 \mathrm{~b}$ shows that the materials are mostly amorphous, and the EDX maps in Fig. $3 \mathrm{c}-\mathrm{e}$ reveal that the sphere is Si rich while the surrounding 

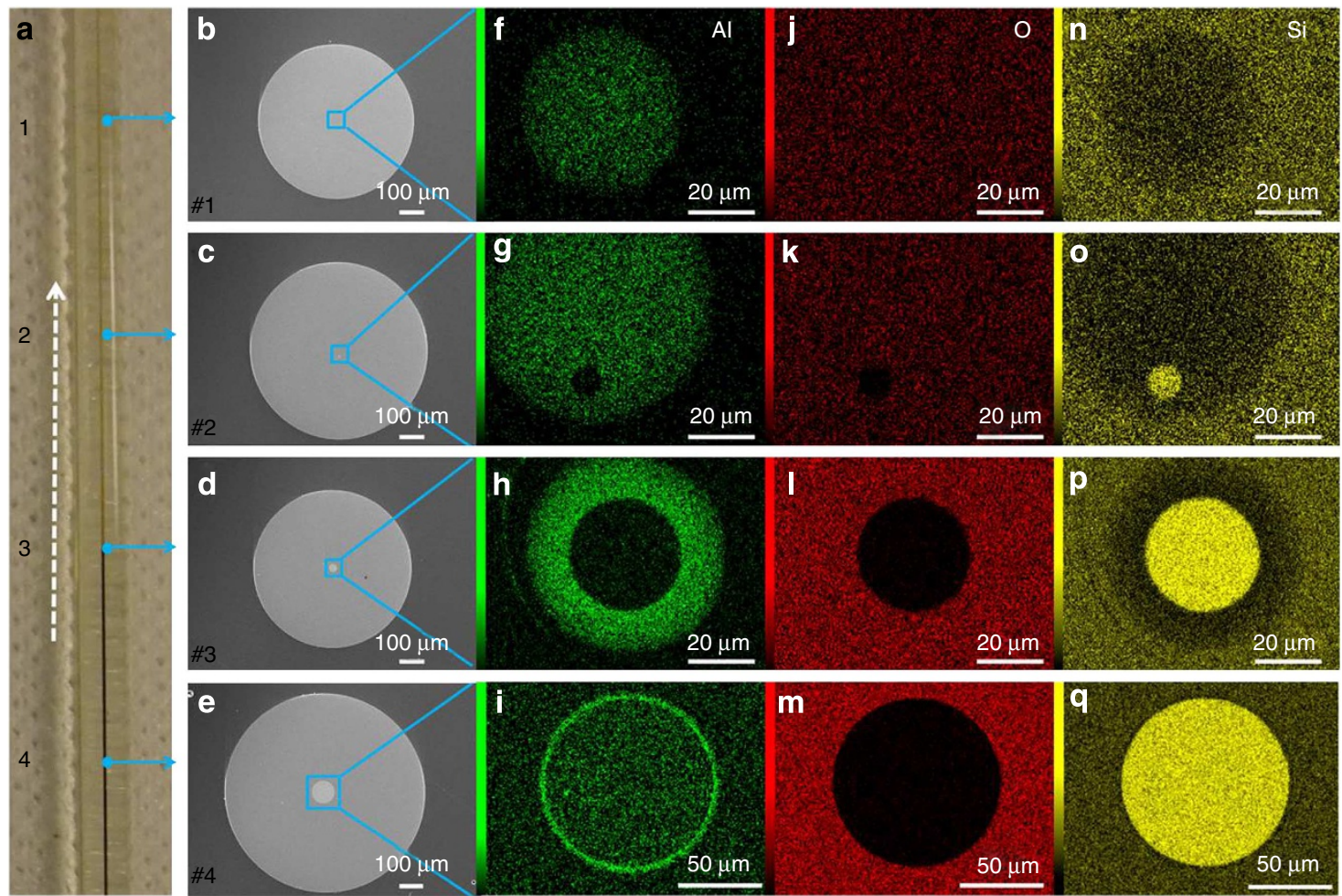

Figure 2 I Transition of fibre core. (a) The optical image of a piece of fibre where a light-yellow core transits to a black core. The white dashed arrow illustrates the fibre drawing direction. (b-e) The SEM images of the cross-sections at the four labelled locations in $\mathbf{a}$. (f-q) The EDX mappings of the marked area in b-e correspondingly. Green, red and yellow denote element aluminium (Al), oxygen (O) and silicon ( $\mathrm{Si}$ ), respectively.
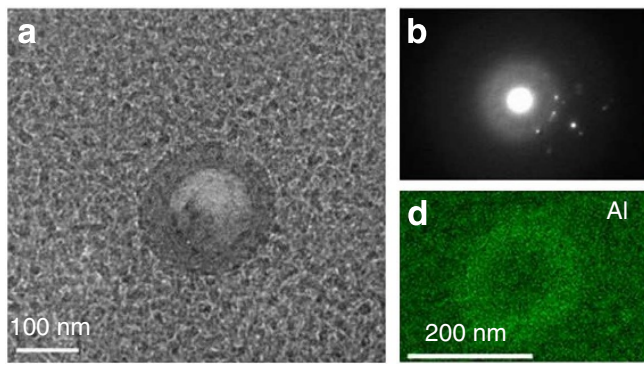

Al
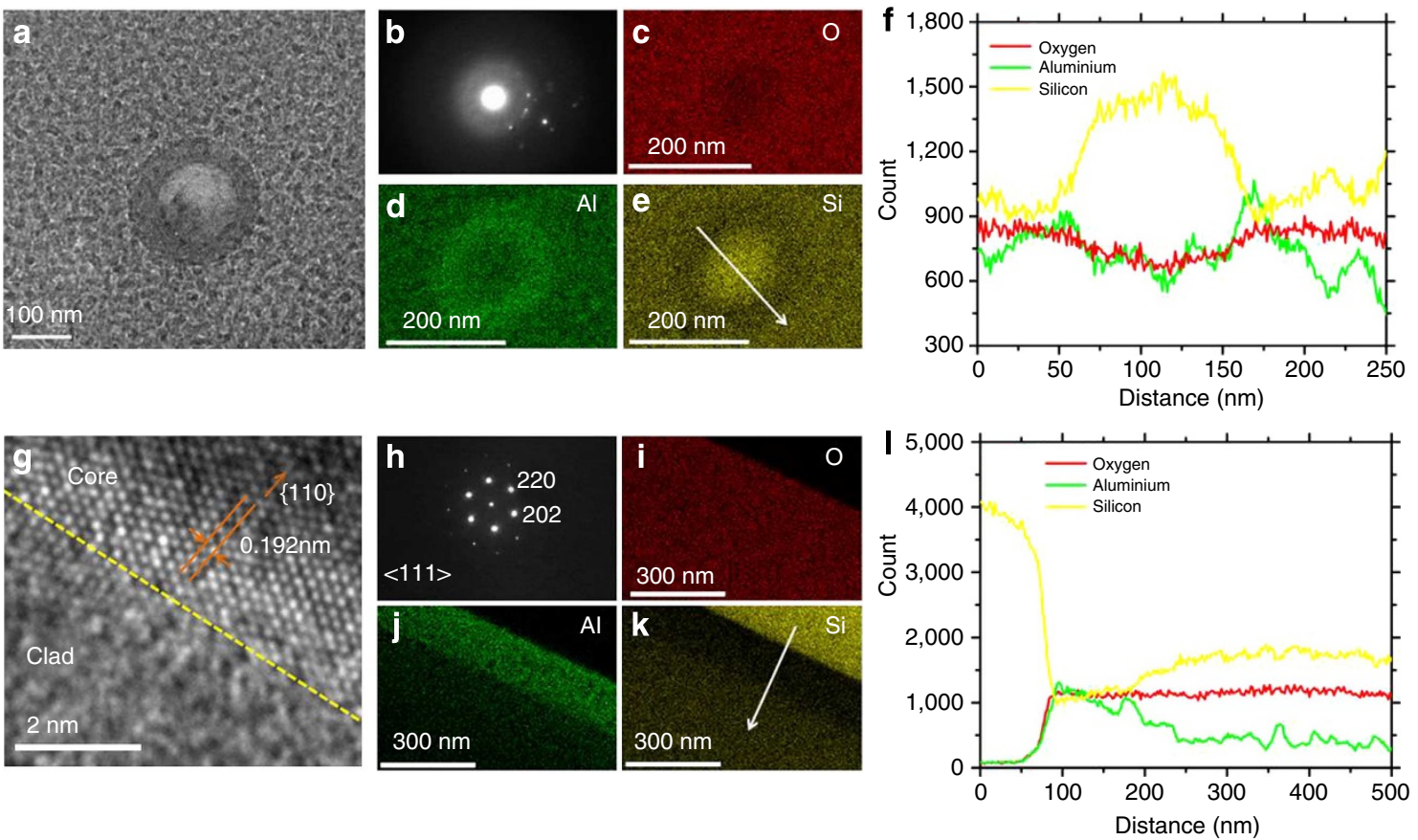

Figure 3 | Nano-scale analyses on fibre core. On the top panel, a-f data are measured inside the core region of the fibre with a yellow core (near location \#2 in Fig. 2a). (a) TEM image, the dot is one of the many dots seen on the sample. (b) The diffraction pattern indicates that the sample is mostly amorphous. (c-e) EDX mappings on $\mathbf{a}$, red, green and yellow denote $\mathrm{O}, \mathrm{Al}$ and $\mathrm{Si}$, respectively. (f) EDX line scan result. The white arrow in $\mathbf{e}$ shows the line scanning path corresponding to $\mathbf{f}$. On the bottom panel, $\mathbf{g}-\mathbf{I}$ data are measured at the core-cladding interface of the fibre with a black core (near location \#4 in Fig. 2a). In $\mathbf{g}$, the yellow dashed line indicates the interface between the crystalline part (core) and the amorphous part (clad). The parallel orange lines are $\{110\}$ faces, and the distance between them is measured $0.192 \mathrm{~nm}$. (h) Taken from crystalline side of the yellow line, and the zone axis has been tilted to $<111>$. (i-k) EDX mappings on $\mathbf{g}$, red, green and yellow denote O, Al and Si, respectively. I, EDX line scan result. The white arrow in $\mathbf{k}$ shows the line scanning path corresponding to $\mathbf{I}$. 
halo is $\mathrm{Al}$ rich. A line scan result across the sphere, as shown in Fig. 3f, confirms this observation. In the case of the black core region (bottom panel), we find distinct properties across the corecladding interface. As shown in Fig. 3g, the dashed yellow line separates a crystalline part from an amorphous part. Figure $3 \mathrm{~h}$ is the diffraction pattern taken on the crystalline side and indicates that the material is crystalline with a diamond cubic structure as viewed along $\langle 111\rangle$ zone axis. The two parallel lines highlighted in Fig. $3 g$ represent $\{110\}$ faces. The distance between the neighbouring faces is $0.192 \mathrm{~nm}$, from which the lattice constant of the crystalline material is calculated to be $0.543 \mathrm{~nm}$. This detailed lattice structure information helps identify that the crystalline material is $\mathrm{Si}$ (Supplementary Fig. 3, Supplementary Note 2). Furthermore, X-ray diffraction (XRD) and Raman spectrum $^{30,31}$ help identify that the Si core wire is polycrystalline, which is similar to that of the core drawn from molten-core method $^{18,24}$ (Supplementary Figs 4 and 5). The EDX mappings shown in Fig. 3i-k clearly demonstrate that the thickness of the Al-rich layer surrounding the Si core is about $100 \mathrm{~nm}$. Finally, the line scan in Fig. 31 demonstrates the purity of $\mathrm{Si}$ in the core region.

Incorporation of crystalline $\mathrm{Si}$ into fibres can enable fibre optoelectronic applications. Current approaches include the molten core drawing or high-pressure CVD with post-annealing process, leading to the demonstration of in-fibre semiconductor devices $^{15,17,23,30}$. Here we characterize the electric performance of the $\mathrm{Si}$ core fabricated by the fibre-draw synthesis method. As shown in Fig. 4a, 20-nm-thick $\mathrm{Al}$ and 150-nm-thick gold ( $\mathrm{Au})$ are deposited on selected locations of a piece of $\mathrm{Si}$ wire (Supplementary Figs 6 and 7, Supplementary Note 3). This piece of $\mathrm{Si}$ wire is taken from the fibre by etching off cladding with $40 \%$ hydrofluoric acid. During the measurement, the 4 -point probe method is used to prevent the possible Shottky junction between the $\mathrm{Si}$ wire and the electrodes. From Fig. $4 \mathrm{~b}$ one could get the resistance per unit distance on the Si wire. Because the Si wire is measured to be $100 \mu \mathrm{m}$ in diameter, the resistivity is calculated to be $0.3 \Omega \mathrm{cm}$ (conductivity $3.4 \mathrm{~S} \mathrm{~cm}^{-1}$ ), which is about the same order as that of the annealed Si core from the high-pressure CVD method and several orders of magnitude smaller than that of intrinsic Si (refs 3,30). Though exact dopant concentration cannot be obtained through the resistivity value alone, it is highly possible that the Si core is doped instead of intrinsic. Further optimization of structure and fabrication process is supposed to achieve better control of material qualities.

Si-core forming mechanism. The mechanism for Si material to appear in the fibre is a chemical reaction between the $\mathrm{Al}$ core and silica cladding. Al has a melting temperature of $660.32^{\circ} \mathrm{C}$, so it melts when fed into the heating zone and keeps its molten state until oxidized ${ }^{32}$. Also as an active reducer, $\mathrm{Al}$ reduces the surrounding silica and produces $\mathrm{Si}$ atoms and $\mathrm{Al}_{2} \mathrm{O}_{3}$ molecules ${ }^{33}$. The drawing temperature of $2,200^{\circ} \mathrm{C}$ is higher than alumina's melting point so that alumina cannot form a shield to protect $\mathrm{Al}$ atoms from oxidizing as it would at room temperature. Without the protection of alumina, the reaction between $\mathrm{Al}$ atoms and silica happens very fast due to two factors: (1) as described by Arrhenius's law, reaction rate relates to temperature exponentially. When the temperature increases by about $2,000 \mathrm{~K}$, the reaction rate is orders of magnitude higher. (2) The largely increased diffusivity at high temperature helps transfer products away from the reaction site at the interface, thus effectively decreasing the products' local concentration and maintaining the chemical reaction. Figure $5 \mathrm{a}$ is a schematic depicting how the chemical reaction happens at the core-cladding interface.
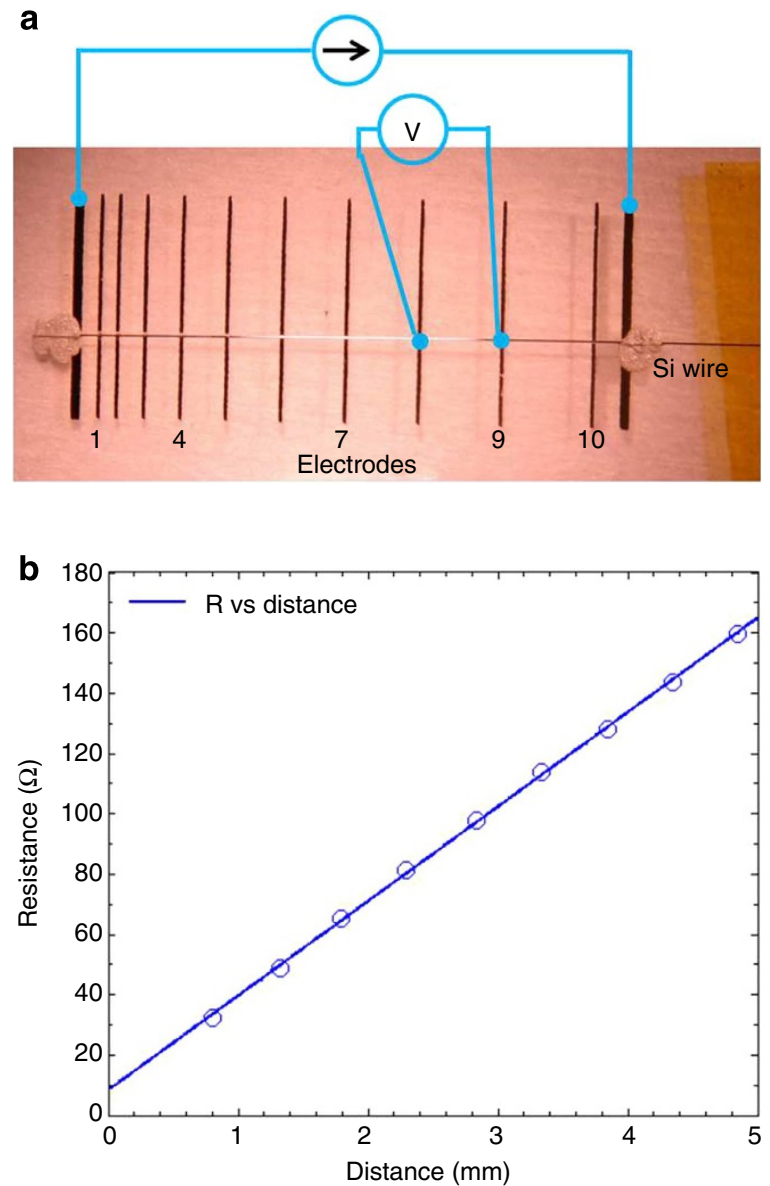

Figure 4 | Si wire electrical characterization. (a) The 4-point probe measurement set-up. A piece of Si wire ( $\sim 3 \mathrm{~cm}$ long) is taken from fibre by etching off the cladding, and then $20 \mathrm{~nm} \mathrm{Al}$ and $150 \mathrm{~nm}$ Au are deposited on selected locations ( 2 outmost electrodes for current source and 10 electrodes for voltage metre contact marked 1 to 10). During the measurement, the probe directly contacts the $\mathrm{Al} / \mathrm{Au}$ layer on top of Si wire. Current is provided from electrodes from two ends, and voltage is measured across neighbourhood electrodes in between the two end electrodes. (b) The resistance across neighbour electrodes are calculated from $\mathbf{a}$ and are plotted against their corresponding distance. Linear regression on the points gives information of Si resistivity.

In addition, although the reduced $\mathrm{Si}$ atoms are mixed with the other product $\mathrm{Al}_{2} \mathrm{O}_{3}$ and not-yet-reacted $\mathrm{Al}$ atoms in the beginning, their solubility properties play an important role in separating them and finally forming a Si core. Most importantly, $\mathrm{Si}$ is completely miscible with $\mathrm{Al}$ in the liquid state, but their solubility with $\mathrm{Al}_{2} \mathrm{O}_{3}$ is comparatively low ${ }^{32,34}$. As more and more $\mathrm{Al}_{2} \mathrm{O}_{3}$ is produced, the excessive $\mathrm{Al}_{2} \mathrm{O}_{3}$ precipitates out of molten Al. Meanwhile, Si stays with molten Al because of their miscibility. As the density of molten $\mathrm{Al}$ and $\mathrm{Si}$ are about $2.4 \mathrm{~g} \mathrm{~cm}^{-3}$ and are smaller than that of molten alumina that is about $2.8 \mathrm{~g} \mathrm{~cm}^{-3}$ (refs 34-36), molten Al-Si floats up and molten alumina sinks to the bottom as shown in Fig. 5b. As the draw and chemical reaction progress, more and more $\mathrm{Al}$ in the $\mathrm{Al}-\mathrm{Si}$ melt gets consumed hence much less $\mathrm{Al}_{2} \mathrm{O}_{3}$ remains in the melt, while $\mathrm{Si}$ keeps accumulating in the Al-Si melt on top of alumina melt. Finally, when $\mathrm{Al}$ is completely consumed in the reaction, a highly pure Si melt is obtained on the top of the core. Further drawing results in a highly pure $\mathrm{Si}$-core fibre. Figure $5 \mathrm{c}-\mathrm{e}$ depicts the above-mentioned core composition change during the draw. 

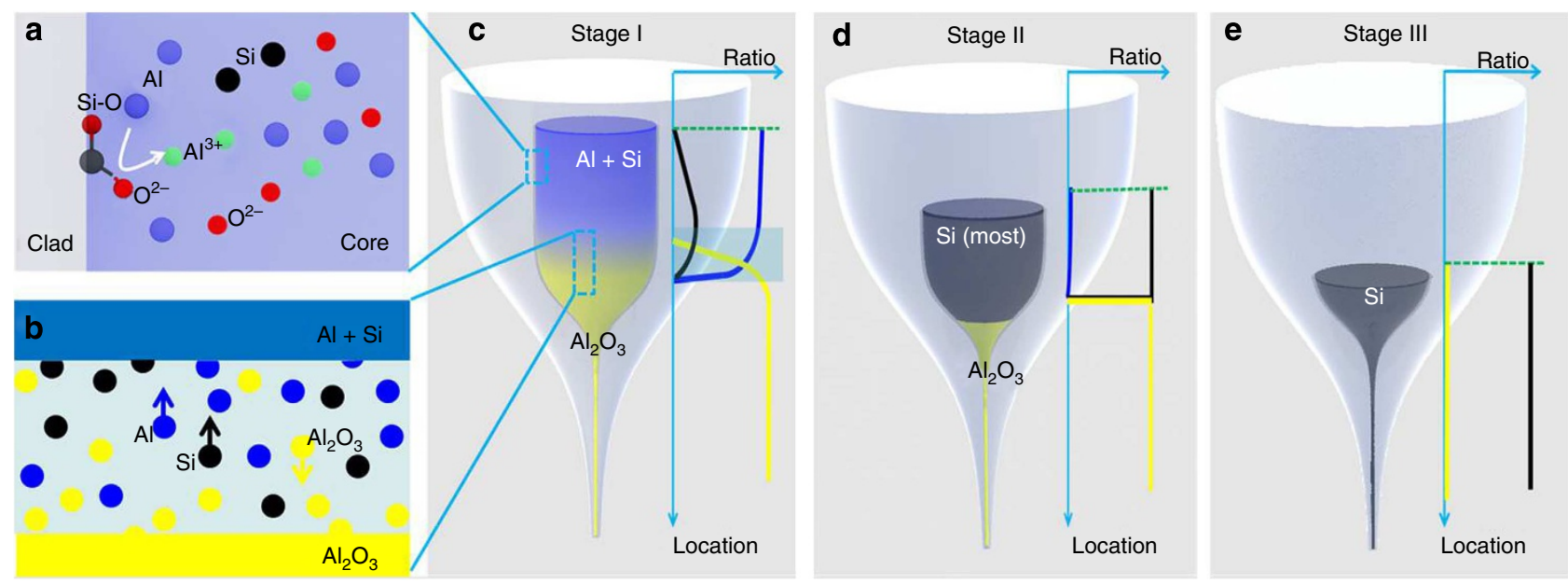

Figure 5 | Si-core forming mechanism in fibre draw. (a) The chemical reaction at the core-cladding interface. Al atoms (blue) break Si-O bond, reduce Si (black), release $\mathrm{O}^{2-}$ ions (red) and change to $\mathrm{Al}^{3+}$ ions (green). All the products of ions and atoms dissolve in the $\mathrm{Al}$ melt and diffuse into the core. (b) The Si atoms (black), because of their miscibility, diffuse and remain in the $\mathrm{Al}$ melt (blue), while ions of $\mathrm{O}$ and $\mathrm{Al}_{\text {form }} \mathrm{Al}_{2} \mathrm{O}_{3}$ molecules (yellow) and precipitate out at the bottom. (c-e) Sketches of the evolution of the core in the heating zone throughout the drawing process. As the draw continues, more and more $\mathrm{Si}$ atoms accumulate in the $\mathrm{Al}-\mathrm{Si}$ melt and eventually one obtains a highly pure $\mathrm{Si}$ melt on top of the $\mathrm{Al}_{2} \mathrm{O}_{3}$ with all the $\mathrm{Al}_{\text {consumed }}$ in the redox reaction (Stage II). Further drawing then results in a highly pure Si-core fibre (Stage III). The diagrams corresponding to each figure demonstrate the ratio of different materials along the core axially. Blue, black and yellow denote $\mathrm{Al}, \mathrm{Si}$ and $\mathrm{Al}_{2} \mathrm{O}_{3}$, respectively. The green dashed line that gets lower and lower during the draw marks the position of the core. The cyan shadows in $\mathbf{b}$ and $\mathbf{c}$ illustrate the location of the transition region.

Solubility not only plays an important role in obtaining a Si-core fibre, but also explains the embedded spheres seen in Fig. $3 \mathrm{a}$ as well. Those Si-rich spheres in alumina are from the small amount of Si that dissolves in molten alumina in the heating zone. As the fibre is pulled out of the furnace and the temperature drops, the solubility of $\mathrm{Si}$ in $\mathrm{Al}_{2} \mathrm{O}_{3}$ decreases. The excess $\mathrm{Si}$ then precipitates out and forms spheres in alumina.

Besides accumulating with the help of miscibility properties, the $\mathrm{Si}$ core is also purified by going through the furnace. Because the preform is fed into the furnace from the top side and the fibre is pulled out of the furnace from the bottom, the difference between the hot furnace temperature and room temperature constructs a temperature profile in which Si melts when entering the hot zone and solidifies when leaving the hot zone. This temperature profile provides the zone melting condition to further purify the $\mathrm{Si}$ core $^{37}$. In addition, $\mathrm{Si}$ is crystallized when it cools down from liquid state after pulling out of furnace ${ }^{38}$.

\section{Discussion}

Our method remains further optimization for future applications. For example, the size of Si-core could be significantly reduced by redesigning the core-clad ratio and adjusting the fibre draw parameters. The redraw technique could also be applied to achieve nanometer-scale $\mathrm{Si}$ wires ${ }^{23}$. Also the thin interface layer that lies between the core and the cladding as seen in Figs $2 \mathrm{i}$ and $3 j$ could be of interest for the potential advantage on oxygen gettering and surface modification for the post-drawing process ${ }^{8,39}$. The smoothness of the $\mathrm{Si}$ is verified in the atomic force scan (AFM) image (Supplementary Fig. 8, Supplementary Note 3). Last but not least, the mechanism of producing Si during fibre drawing is also applicable to other reductive metals, for example, Titanium (Ti) or Calcium $(\mathrm{Ca})$, which could reduce $\mathrm{Si}$ because of their more negative reduction potentials ${ }^{40}$.

To conclude, we demonstrate a new way to fabricate a high-quality crystalline Si-core fibre by thermally drawing a preform consisting of low-cost metal. The methods described herein set the stage for extending the materials and architecture selections with fibre drawing, thus paving the way towards more sophisticated fibre-based devices.

\section{Methods}

Preform fabrication and fibre drawing. For the preform preparation we use the fused-quartz tubes and rods supplied by Technical Glass Products and the Al wire from Alfa Aesar. The fused-quartz capillary fibre $(300 \mu \mathrm{m}$ inner diameter and $600 \mu \mathrm{m}$ outer diameter) is from Molex Incorporated (product \# TSP300794). The coating material on the capillary fibre is burned with flame and cleaned up with isopropanol.

The 0.25 - $\mathrm{mm}$-diameter $\mathrm{Al}$ wire is first inserted into the capillary fibre and then put into a fused-quartz tube with an inner diameter of $1 \mathrm{~mm}$ and outer diameter of $6 \mathrm{~mm}(1 \times 6$ tube). Two 1-mm-diameter fused-quartz rod are also inserted into the $1 \times 6$ tube, one on top of the capillary fibre and the other in the bottom of the capillary fibre. The whole assembly is put into a fused-quartz tube with an inner diameter of $6 \mathrm{~mm}$ and outer diameter of $12 \mathrm{~mm}$. With the help of propane flame, one end of the structure is fused and the whole assembly is pumped to vacuum and the other end is fused under vacuum.

The fibre drawing process starts at the temperature of $2,150{ }^{\circ} \mathrm{C}$ and the drawing process continues at $2,200^{\circ} \mathrm{C}$. The preform is fed into the furnace from top with a speed of $1 \mathrm{~mm} \mathrm{~min}^{-1}$, and the fibre is pulled out from the bottom end of the furnace with a speed of $660 \mathrm{~mm} \mathrm{~min}^{-1}$. The fibre is further coated with coating material, which is called 'DSM Desotech 3471-3-14(941-314) Desolite single coat coating', and cured by an in-line ultraviolet coating system.

Characterization instruments and methods. The SEM images and related EDX mappings on cross-sections of the fibres are taken with the JEOL 6010LA SEM. The TEM samples are prepared in the Helios Nanolab 600 Dual Beam Focused Ion Beam Milling System, and the process of preparing a TEM sample is detailed in the Supplementary Information (Supplementary Fig. 1, Supplementary Note 1). The TEM images and related EDX scans are taken using the JEOL 2010 FEG Analytical Electron Microscope. The Raman spectrum is taken in the Horiba Jobin Yvon LabRAM Model HR800. The XRD spectrum is taken in Rigaku SmartLab with a parallel-beam optics and capillary-sample set-up. The electrical characterization of the Si wire is on a 4-point stage using the Hewlett-Packard (HP) 4155A Semiconductor Parameter Analyser. The surface smoothness of the Si wire is characterized with Digital Instruments Veeco Dimension 3100 AFM, and the data are processed in the Nanoscope Analysis software (v1.40) from Bruker Corporation.

\section{References}

1. Dresselhaus, G., Kip, A. \& Kittel, C. Cyclotron resonance of electrons and holes in silicon and germanium crystals. Phys. Rev. 98, 368-384 (1955).

2. Klement, W., Willens, R. H. \& Duwez, P. Non-crystalline structure in solidified gold-silicon alloys. Nature 187, 869-870 (1960).

3. Seto, J. Y. W. The electrical properties of polycrystalline silicon films. J. Appl. Phys. 46, 5247-5254 (1975).

4. Lin, V. S.-Y., Motesharei, K., Dancil, K.-P. S., Sailor, M. J. \& Ghadiri, M. R. A porous silicon-based optical interferometric biosensor. Science 278, 840-843 (1997). 
5. Pavesi, L., Dal Negro, L., Mazzoleni, C., Franzò, G. \& Priolo, F. Optical gain in silicon nanocrystals. Nature 408, 440-444 (2000).

6. Vlasov, Y. A., Bo, X. Z., Sturm, J. C. \& Norris, D. J. On-chip natural assembly of silicon photonic bandgap crystals. Nature 414, 289-293 (2001).

7. Cui, Y. \& Lieber, C. M. Functional nanoscale electronic devices assembled using silicon nanowire building blocks. Science 291, 851-853 (2001).

8. Xiang, J. et al. Ge/Si nanowire heterostructures as high-performance field-effect transistors. Nature 441, 489-493 (2006).

9. Tian, B. et al. Coaxial silicon nanowires as solar cells and nanoelectronic power sources. Nature 449, 885-889 (2007).

10. Atwater, H. A. \& Polman, A. Plasmonics for improved photovoltaic devices. Nat. Mater. 9, 205-213 (2010).

11. Foster, M. A. et al. Silicon-chip-based ultrafast optical oscilloscope. Nature 456, 81-84 (2008).

12. Leuthold, J., Koos, C. \& Freude, W. Nonlinear silicon photonics. Nat. Photon. 4, 535-544 (2010).

13. Reed, G. T., Mashanovich, G., Gardes, F. Y. \& Thomson, D. J. Silicon optical modulators. Nat. Photon. 4, 518-526 (2010).

14. Liu, A. et al. A high-speed silicon optical modulator based on a metal-oxidesemiconductor capacitor. Nature 427, 615-618 (2004).

15. He, R. et al. Integration of gigahertz-bandwidth semiconductor devices inside microstructured optical fibres. Nat. Photon. 6, 174-179 (2012).

16. Lin, H. et al. Twisted aligned carbon nanotube/silicon composite fiber anode for flexible wire-shaped lithium-ion battery. Adv. Mater. 26, 1217-1222 (2014).

17. He, R. et al. Silicon p-i-n junction fibers. Adv. Mater. 25, 1461-1467 (2013).

18. Martinsen, F. a. et al. Silicon-core glass fibres as microwire radial-junction solar cells. Sci. Rep. 2, 591 (2014).

19. Won, D.-J. et al. All-optical modulation of laser light in amorphous siliconfilled microstructured optical fibers. Appl. Phys. Lett. 91, 161112 (2007).

20. Healy, N. et al. Extreme electronic bandgap modification in laser-crystallized silicon optical fibres. Nat. Mater. 13, 1122-1127 (2014).

21. Mehta, P., Healy, N., Day, T. D., Badding, J. V. \& Peacock, A. C. Ultrafast wavelength conversion via cross-phase modulation in hydrogenated amorphous silicon optical fibers. Opt. Express 20, 26110-26116 (2012).

22. Peacock, A. C., Mehta, P., Horak, P. \& Healy, N. Nonlinear pulse dynamics in multimode silicon core optical fibers. Opt. Lett. 37, 3351-3353 (2012).

23. Gumennik, A. et al. Silicon-in-silica spheres via axial thermal gradient in-fibre capillary instabilities. Nat. Commun. 4, 2216 (2013).

24. Ballato, J. et al. Silicon optical fiber. Opt. Express 16, 18675-18683 (2008).

25. Sazio, P. J. a. et al. Microstructured optical fibers as high-pressure microfluidic reactors. Science 311, 1583-1586 (2006).

26. Orf, N. D. et al. Fiber draw synthesis. Proc. Natl Acad. Sci. 108, 4743-4747 (2011).

27. Morris, S. et al. Reactive molten core fabrication of silicon optical fiber. Opt. Mater. Express 1, 1141-1149 (2011).

28. Hou, C. et al. Direct atomic-level observation and chemical analysis of $\mathrm{ZnSe}$ synthesized by in situ high-throughput reactive fiber drawing. Nano Lett. 13 975-979 (2013).

29. Wang, D. \& Shi, Z. Aluminothermic reduction of silica for the synthesis of alumina-aluminum-silicon composite. J. Mater. Synth. Process 9, 241-246 (2001).
30. Finlayson, C. E., Amezcua-Correa, a., Sazio, P. J. a., Baril, N. F. \& Badding, J. V. Electrical and Raman characterization of silicon and germanium-filled microstructured optical fibers. Appl. Phys. Lett. 90, 132110 (2007).

31. Lagonigro, L. et al. Low loss silicon fibers for photonics applications. Appl. Phys. Lett. 96, 041105 (2010).

32. Okamoto, H. \& Massalski, T. B. in Desk Handbook: Phase Diagrams for Binary Alloys (ed. Okamoto, H.) xxxix-xliii (ASM International, 2000).

33. Standage, A. E. \& Gani, M. S. Reaction between vitreous silica and molten aluminum. J. Am. Ceram. Soc. 50, 101-105 (1967).

34. Hatch, J. E. Aluminum: Properties and Physical Metallurgy vol. 1 (ASM International, 1984).

35. Inatomi, Y., Onishi, F., Nagashio, K. \& Kuribayashi, K. Density and thermal conductivity measurements for silicon melt by electromagnetic levitation under a static magnetic field. Int. J. Thermophys. 28, 44-59 (2007).

36. Aksay, I. A., Pask, J. A. \& Davis, R. F. Densities of $\mathrm{SiO} 2-\mathrm{Al} 2 \mathrm{O} 3$ melts. J. Am. Ceram. Soc. 62, 332-336 (1979).

37. Pfann, W. G. Zone Melting: This technique offers unique advantages in purification and in control of composition in various substances. Science 135, 1101-1109 (1962).

38. Olson, G. L. \& Roth, J. A. Kinetics of solid phase crystallization in amorphous silicon. Mater. Sci. Rep. 3, 1-77 (1988).

39. Nordstrand, E. F., Dibbs, A. N., Eraker, A. J. \& Gibson, U. J. Alkaline oxide interface modifiers for silicon fiber production. Opt. Mater. Express 3, 651-657 (2013).

40. Atkins, P. W. \& de Paula, J. Physical Chemistry (W.H. Freeman, 2002).

\section{Acknowledgements}

This work was supported in part by the MIT MRSEC through the MRSEC Programme of the National Science Foundation under award number DMR-0819762 and also was supported in part by the US Army Research Laboratory and the US Army Research Office through the Institute for Soldier Nanotechnologies, under contract number W911NF-13-D-0001.

\section{Author contributions}

C.H. and L.W. fabricated fibre, S.T. prepared TEM samples, C.H., S.T. and X.J. conducted TEM analysis, C.H. and X.Z. provided electrical characterization, C.H. conducted other experiments, C.H., J.J. and Y.F. discussed the Si-core forming mechanism and C.H. and Y.F. wrote the manuscript.

\section{Additional information}

Supplementary Information accompanies this paper at http://www.nature.com/ naturecommunications

Competing financial interests: The authors declare no competing financial interests

Reprints and permission information is available online at http://npg.nature.com/ reprintsandpermissions/

How to cite this article: Hou, C. et al. Crystalline silicon core fibres from aluminium core preforms. Nat. Commun. 6:6248 doi: 10.1038/ncomms7248 (2015). 